\title{
Le repérage des patients en situation de précarité par les médecins généralistes
}

The identification of patients in precarious situations by general practitioners

Katia Mazalovic, Claire Zabawa, Pierre-Henri Roux-Levy et Maryse Gaimard

\section{OpenEdition}

1 Journals

Édition électronique

URL : https://journals.openedition.org/popvuln/1071

DOI : $10.4000 /$ popvuln. 1071

ISSN : 2650-7684

Éditeur

LIR3S - Laboratoire Interdisciplinaire de Recherche "Sociétés Sensibilités Soin" (UMR 7366 CNRS-uB)

Édition imprimée

Date de publication : 1 mars 2019

Pagination : 157-179

ISBN : 978-2-918173-26-7

ISSN : 2269-0182

Référence électronique

Katia Mazalovic, Claire Zabawa, Pierre-Henri Roux-Levy et Maryse Gaimard, « Le repérage des patients en situation de précarité par les médecins généralistes », Populations vulnérables [En ligne], 5 | 2019, mis en ligne le 26 mai 2020, consulté le 26 février 2022. URL : http://journals.openedition.org/popvuln/ 1071 ; DOI : https://doi.org/10.4000/popvuln.1071

Ce document a été généré automatiquement le 26 février 2022.

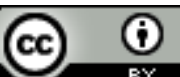

Les contenus de la revue Populations vulnérables sont mis à disposition selon les termes de la Licence Creative Commons Attribution 4.0 International 


\section{Le repérage des patients en situation de précarité par les médecins généralistes}

The identification of patients in precarious situations by general practitioners

Katia Mazalovic, Claire Zabawa, Pierre-Henri Roux-Levy et Maryse Gaimard

\section{Précarité : définitions et contexte}

1 Le terme " précarité » est très utilisé, dans des registres variés, lui attribuant de fait un caractère polysémique. Cette grande variabilité des sens relatifs au mot "précarité » est inévitablement associée à des difficultés de traduction, limitant ainsi les possibilités de comparaison internationales (Barbier, 2005; Cingolani, 2006). Il semble donc important de définir ce terme dans un premier temps. Sur un plan étymologique, le mot précarité est issu du terme latin precarius qui signifie « qui s'obtient par la prière ». Ce terme est donc porteur d'un double sens, juridique et religieux, et implique un principe de révocabilité. La définition proposée par Littré dès 1880 était plus précise et désignait une prérogative s'exerçant "par permission " sinon "par tolérance » et supposait également une "dépendance» (Littré, 1880). Plus tard, le dictionnaire Larousse donne la définition et les exemples suivants, confirmant cette notion de révocabilité et d'instabilité : É tat de ce qui est précaire. Qui n'existe ou ne s'exerce que par une autorisation révocable : poste précaire. Qui n'offre nulle garantie de durée, de stabilité, qui peut toujours être remis en cause : santé précaire, emploi précaire. Qui est d'une sécurité douteuse : un abri précaire». Dans les années 1990, une définition socio-administrative de la précarité a été élaborée, basée avant tout sur la situation d'un individu par rapport à l'emploi (ministère des Affaires sociales et de l'Intégration, 1992 ; Sass, 2006a). Le Haut conseil de santé publique, dans son rapport sur «la grande pauvreté et la précarité 
économique ", a produit une définition plus complexe, de par son caractère multidimensionnel :

état d'instabilité sociale caractérisée par la perte d'une ou plusieurs des sécurités, notamment celle de l'emploi, permettant aux personnes et aux familles d'assumer leurs responsabilités professionnelles, familiales et sociales et de jouir de leurs droits

fondamentaux » (Wrezinski,

1987). Cette dernière définition permet d'inclure d'autres dimensions qu'une dimension purement économique, en différenciant la précarité de la pauvreté. La précarité est une notion globale, tenant compte des différentes caractéristiques d'un individu (biologiques, psychologiques et sociales). La précarité peut fluctuer dans le temps, en fonction de la trajectoire sociale d'un individu. La précarité ainsi définie correspond donc à un état potentiellement réversible, pouvant conduire à des situations d'exclusion sociale ou d'extrême pauvreté, qui, quant à elles, ne sont plus réversibles. Encore plus récemment, Furtos a défini la précarité comme une absence (ou une perte) d'objet social (Furtos, 2000). Ici, un objet social est défini comme «quelque chose de concret comme le travail, l'argent, le logement, la formation ou les diplômes", constituant, pour un individu donné, une « sécurité de base » lui permettant d'assurer son statut social et de faire face à ses besoins. L'objet social défini ici tient sa valeur de la sécurité qu'il apporte à l'individu, mais aussi de son aspect social, par le statut qu'il accorde à l'individu au sein de la société (Furtos, 2000 ; Furtos, 2007).

\section{La précarité : un repérage « objectif » difficile}

2 En pratique, la complexité inhérente à cette notion de précarité rend son repérage plus difficile. En effet, quand (et à partir de quand) peut-on dire qu'un individu est précaire ou non? Et sur la base de quel(s) élément(s) ? Lorsqu'il est nécessaire d'objectiver la précarité, comme par exemple dans le domaine de la recherche, la procédure se complexifie encore. Face à cette problématique d'évaluation de la précarité, de nombreuses équipes ont développé différents scores (ou échelles).

3 Parmi les évaluations disponibles de la précarité, certaines sont dites "écologiques ", c'est-à-dire utilisables sur une population, de façon collective (souvent, une zone géographique donnée), à partir de données agrégées, et non sur un individu isolé (Carstairs, 1989 ; Jordan, 2004 ; ministère des Affaires sociales et de l'Intégration, 1992). Celles-ci utilisent fréquemment des indicateurs relatifs aux conditions de vies matérielles, à certains équipements disponibles ou non. Ces échelles ont une utilité dans certaines études épidémiologiques, mais ne rendent pas compte du caractère multidimensionnel de la précarité.

D'autres évaluations sont dites "individuelles ", c'est-à-dire qu'elles permettant de statuer sur un individu donné. Parmi celles-ci, en excluant les scores non validés (Robert, 2013) et ceux ne faisant référence qu'à la dimension matérielle de la précarité, peu de scores restent disponibles (Adams, 2005; Pascal, 2006). Une équipe néozélandaise a développé le score "NZiDep». Ce dernier semble très difficilement transposable au contexte européen (ou français) en raison de la spécificité de certains items utilisés qui semblent peu pertinents dans notre environnement (par exemple, un item interroge sur la nécessité pour une personne de porter des chaussures trouées) (Salmond, 2006). Il en est de même pour le score turc, «FWID» (Eroğlu, 2007). En Suisse, c'est le score «DIPCare-Q» qui a été développé. Le contexte économique et 
social suisse étant relativement différent du contexte français, la transférabilité de ce score est également limitée. Par ailleurs, ce dernier est un score continu, ne permettant pas de catégoriser les individus en "précaires » ou «non précaires» (Vaucher, 2012). Enfin, le score " Handicap social », développé en France, semble peu adapté à un usage répétitif en raison de sa longueur (111 items) et de sa validation effectuée essentiellement en milieu hospitalier (Castiel, 2009).

Le score EPICES (évaluation de la précarité et des inégalités de santé dans les centres d'examens de santé) a été construit en 1998, selon une méthodologie robuste et a été validé sur une population importante dans les centres d'examens de santé français (Moulin, 2005 ; Sass, 2006b). Ce score peut être utilisé comme une variable continue ou comme une variable discrète, permettant dans ce cas de catégoriser les individus en " précaires » ou « non précaires » (selon que leur score dépasse le seuil de 30 ou non) (Labbe, 2007 ; Labbe, 2015 ; Sass, 2006b). Il tient compte du caractère multidimensionnel de la précarité, en permettant l'identification d'une population non différenciée par des marqueurs économiques (Sass, 2006b).

\section{Relation entre précarité et santé}

6 Sur un plan sanitaire, les personnes en situation de précarité sont particulièrement concernées par les conséquences des inégalités sociales de santé. Cette catégorie de personnes, dites " précaires ", est donc une population particulièrement vulnérable sur le plan sanitaire (Collet, 2001). Les personnes qualifiées de précaires (d'après différents scores) présentent des indicateurs de santé plus mauvais, notamment en termes de mortalité, de morbidité ou d'état de santé perçu, que le reste de la population (Labbe, 2015). La précarité, selon le score EPICES, est associée à de nombreux indicateurs de santé péjoratifs (Moulin, 2006): consommation de tabac élevée, consommation journalière d'alcool plus élevée, en accord avec les résultats des valeurs biologiques corrélées à cette consommation, obésité chez les femmes, absence de suivi gynécologique (pour les dépistages de base), non-suivi médical chez les hommes.

7 Ces personnes en situation de précarité ne sont pas rares. En effet, selon le peu de données disponibles, $20 \%$ de la population française pourrait être considérée comme précaire (ministère de l'Emploi et de la Solidarité, 1998 ; Robert, 2013). Ainsi, loin de constituer des exceptions, les personnes précaires, plus vulnérables sur un plan sanitaire, constituent une population sur laquelle des efforts de préventions spécifiques sont nécessaires. Le médecin généraliste semble être l'acteur le plus stratégique pour renforcer les efforts de prévention chez le groupe à risque que constituent les personnes précaires. En effet, la médecine générale (ou soins de santé premiers) constitue le premier niveau de soins offert par le système de santé français. Sa proximité avec les patients, son accessibilité, son coût plus faible (en comparaison avec les spécialités d'organes ou secondaires) constituent ses principaux avantages.

8 Cet enjeu d'équité des soins (faire plus avec ceux qui ont besoin de plus) nécessite, en pratique, une identification des personnes précaires. Ainsi, avant d'évaluer la possibilité (et l'efficience) d'un renforcement des efforts de prévention chez les personnes précaires, il convient de s'interroger sur la possibilité d'une identification préalable de la précarité chez les personnes accessibles à ces efforts, c'est-à-dire chez les personnes consultant en médecine générale. 
9 Dans la suite de cet article, nous proposons d'évaluer la concordance entre le repérage de la précarité par les médecins généralistes et le repérage par le score le mieux évalué, à savoir le score EPICES.

\section{Méthode de recherche}

\section{1) Type d'étude}

10 Une étude quantitative, observationnelle, transversale et multicentrique a été réalisée en Bourgogne entre 2013 et 2014. Son objectif principal était l'évaluation de la concordance entre le repérage de la précarité effectué instinctivement ou spontanément par les médecins généraliste auprès des patients les consultant, et un repérage effectué à l'aide d'un score, le score EPICES. Les objectifs secondaires étaient d'étudier les variables associées à la concordance et à la discordance entre l'évaluation spontanée du médecin généraliste et le score EPICES.

11 En s'appuyant sur les estimations disponibles quant à la prévalence de la précarité en France (ministère de l'Emploi et de la Solidarité, 1998), le nombre de personnes à inclure dans l'étude était de 200.

\section{2) Population d'étude et recueil de données}

12 Les médecins généralistes participants ont été recrutés parmi les médecins généralistes maîtres de stages universitaires de Bourgogne, c'est-à-dire auprès des médecins généralistes agréés pour recevoir des étudiants en médecine de septième et huitième années. Ces médecins généralistes volontaires devaient, afin de pouvoir organiser le recueil de données dans leur cabinet avec leur étudiant, pouvoir mettre à disposition une pièce fermée au sein du cabinet médical. Cette nécessité « pratique » a limité de fait le nombre de médecins généralistes pouvant participer. Les caractéristiques des médecins participants ont été recueillies (âge, sexe, zone d'installation, durée d'installation, opinion concernant leur rôle dans le repérage de la précarité et utilisation des outils d'aide au repérage).

Pour des raisons de faisabilité, les inclusions des patients ne devaient pas perturber l'apprentissage des étudiants en stage et devaient être concentrées sur certaines périodes de stage. En tenant compte de ces contraintes, trois patients devaient être inclus chaque jour, par l'étudiant en stage, sur de courtes périodes. Afin de limiter le biais de sélection, les patients à inclure n'étaient pas choisis par les médecins généralistes ou les étudiants en stage. Ainsi, les patients étaient ciblés par une liste de randomisation, identifiant les patients en fonction de leur ordre de passage en consultation sur l'agenda du médecin. Ces listes étaient établies à l'avance, à l'aide d'un logiciel de randomisation, en se servant du nombre moyen de consultations effectuées chaque jour par un praticien donné. Après la consultation d'un patient «ciblé » par la liste de randomisation, il lui était proposé de participer à notre étude. Les patients acceptant de participer étaient ensuite conduits dans une pièce différente du bureau de consultation du médecin généraliste, afin d'y effectuer le recueil de données sur un formulaire papier. Ce recueil comprenait le score EPICES ainsi que des données sociodémographiques (âge, sexe, personne vivant seule ou en couple, niveau d'études, profession et catégorie sociale, emploi pérenne ou non, personne bénéficiaire de la 
CMU, personne titulaire d'une assurance maladie complémentaire ou mutuelle). Pendant ce temps, le médecin généraliste effectuait, de façon indépendante et en aveugle, son évaluation du statut du patient vis-à-vis de la précarité. La question était libellée de la façon suivante: "selon vous, le patient que vous venez de recevoir en consultation est-il en situation de précarité ? Oui/Non »). Une évaluation du degré de certitude du médecin était fournie en dessous de cette évaluation de la précarité (échelle allant de 0 à 10 selon que le médecin était complètement incertain ou certain de son évaluation).

patients inclus dans l'étude devaient répondre aux critères d'inclusion suivants être ciblé par la liste de randomisation, être majeur et en état de comprendre les consignes données. Les patients sous tutelle, curatelle ou sauvegarde de justice étaient exclus de l'étude. Les patients vus en visite à domicile étaient également exclus de l'étude.

15 Les refus de participer ont été colligés anonymement, quelle qu'en fût la raison invoquée, afin de rendre compte d'un éventuel biais de sélection.

\section{3) Critères de jugement}

Score EPICES était utilisé pour catégoriser les patients participants en précaires ou non précaires en fonction de leur score. Les participants ayant un score EPICES supérieur à 30/100 étaient considérés comme précaires.

17 Les médecins généralistes devaient estimer la précarité de leurs patients en répondant par oui ou non à la question suivante : selon vous, votre patient est-il en situation de précarité ? Le médecin devait également évaluer son degré de certitude sur une échelle de 0 à 10 ( 0 : pas certain du tout de son évaluation, et 10 : certain de son évaluation).

18 Les autres variables recueillies étaient: l'âge, le mode de vie (seul ou en couple), le niveau d'étude (inférieur au baccalauréat, niveau baccalauréat, supérieur au baccalauréat), la profession et catégorie socio-professionnelle selon l'INSEE (Institut national de la statistique et des études économiques) (agriculteurs exploitants; artisans; commerçants et chefs d'entreprise; cadres et profession intellectuelles supérieures; professions intermédiaires; employés; ouvriers; retraités; autres personnes sans activité professionnelle), la pérennité de l'emploi (emploi pérenne type contrat à durée indéterminée $v s$ emploi non pérenne, type contrat à durée déterminée), la situation matrimoniale (personne seule vs en couple), le fait de bénéficier de la CMU (Couverture maladie universelle, qui est une assurance santé complémentaire au régime obligatoire, attribuée sous conditions de ressources de façon à dispenser une personne de l'avance des frais liés à ses soins de santé, du ticket modérateur et de la participation forfaitaire aux soins de santé), le fait de bénéficier ou non d'une mutuelle.

\section{4) Analyse des données}

19 Les données anonymisées recueillies ont été saisies puis analysées à l'aide du logiciel $\mathrm{R}$ (version 3.1.1).

20 Conformément à la règlementation, une déclaration auprès de la Commission nationale de l'informatique et des libertés (CNIL) a été effectuée $\left(\mathrm{n}^{\circ} 1747242 \mathrm{v} 0\right)$. Le consentement écrit de chaque patient a été recueilli, après explication orale des implications de notre étude observationnelle. Un score de concordance Kappa de Cohen

Populations vulnérables, 5 | 2019 
entre le score EPICES et le repérage spontané effectué par le médecin généraliste a été calculé. Des tests d'association d'une variable ont été effectués en cas de discordance ou de concordance entre l'évaluation du médecin et le score EPICES, à l'aide des tests usuels (Fisher, Chi 2 en fonction des effectifs attendus pour les variables qualitatives, ou test de Student pour les variables quantitatives). Le seuil de signification a été fixé à 0,05 pour toutes les analyses. Une analyse multivariée des variables associées à la concordance a été effectuée par régression logistique pas à pas.

\section{Résultats et discussion}

\section{1) Population des médecins recrutant les patients}

21 Parmi les 90 médecins généralistes maîtres de stage recevant des étudiants de septième et huitième années, 32 médecins étaient volontaires pour participer et avaient des locaux compatibles avec le recueil des données. Notre population d'étude étant constituée uniquement à partir de ces cabinets médicaux, il convient de s'interroger sur la représentativité des médecins généralistes maîtres de stage, par rapport à la population des médecins généralistes dans son ensemble. Des études ont montré que ces deux populations présentaient des différences (Berkhout, 2017). D'après leurs données, les maîtres de stages diffèrent essentiellement par leur âge (ils sont plus jeunes), par un exercice mixte plus fréquent, et par un exercice plus souvent localisé en milieu rural. Les patients des maitres de stage sont moins souvent bénéficiaires de la CMU. Ainsi, notre échantillon est donc biaisé par ce recrutement effectué uniquement parmi les maîtres de stages, pour des raisons de faisabilité. Ces différences semblent toutefois ténues, même si notre mode d'échantillonnage était donc susceptible de limiter la proportion de patients précaires inclus dans notre étude.

Parmi les médecins généralistes volontaires pour participer, 43,7 \% étaient des femmes. L'âge moyen de ces médecins était de 53,1 ans et ils étaient installés dans leur cabinet depuis 21,8 ans en moyenne. Ils recevaient des étudiants en médecine en stage au sein de leur cabinet depuis 7,3 années en moyenne. Les cabinets médicaux de ces médecins étaient situés en zone urbaine dans $37,5 \%$ des cas, en zone semi-rurale dans $34,8 \%$ des cas, et en zone rurale pour $28,1 \%$ d'entre eux. La surreprésentation des médecins exerçant en milieu rural peut en partie s'expliquer par le recrutement effectué parmi les généralistes maitres de stage. Toutefois, il est possible que le fait de devoir bénéficier de locaux permettant le recueil de données par l'interne, en aveugle du médecin, ait limité l'inclusion de médecins exerçant en zone urbaine, où les surfaces des locaux professionnels sont inférieures.

Les médecins participants estimaient pour 93,7\% d'entre eux que le repérage de la précarité faisait partie de leur rôle de médecin traitant, et $28,1 \%$ déclaraient l'intégrer systématiquement dans leur pratique. Une différence notable a donc été retrouvée, avec d'un côté un intérêt déclaré pour la thématique de la précarité, intégrée au rôle du médecin généraliste, et un repérage en pratique peu réalisé. Les causes de cette ambivalence n'ont pas été identifiées ici. Néanmoins, l'hypothèse de difficultés dans la mise en œuvre pratique de ce repérage peut être formulée.

L'exploration des moyens de repérage de la précarité connus par les médecins généralistes participants à l'étude montrait que seulement $3(9,4 \%)$ médecins participants connaissaient et nommaient spontanément le score EPICES comme étant 
un outil pour repérer la précarité. Parmi les critères utilisés en pratique pour estimer ou identifier la précarité de leurs patients, 27 (83,9\%) médecins déclaraient prendre en compte la profession, $25(77,4 \%)$ prenaient en compte l'aspect vestimentaire du patient, 29 (90,3\%) considéraient la situation matrimoniale, 29 (90,3\%) intégraient le fait de bénéficier de la CMU et 22 (67,7\%) appréciaient le niveau d'études de leurs patients. Les éléments de repérage évoqués par les médecins participants pouvaient être classés en deux catégories. La première était constituée d'éléments « objectivables » comme la profession, le niveau d'études ou le fait de bénéficier de la $\mathrm{CMU}$ et semble plutôt faire référence à des critères économiques permettant de déduire ou d'approximer dans une certaine mesure un niveau de revenus pour la personne concernée. La seconde catégorie, quant à elle, était constituée d'éléments moins objectivables comme la tenue vestimentaire et/ou la situation matrimoniale. L'utilisation effective de ces éléments semble plus complexe, tant dans leurs définitions (ou seuils) que dans leur fidélité à retranscrire la précarité. En pratique, ces critères sont probablement utilisés en complément d'autres critères dans l'évaluation de la position sociale d'un patient.

Enfin, selon les médecins interrogés, le fait d'identifier la vulnérabilité sociale de leurs patients influençait la prise en charge pour $18(54,8 \%)$ d'entre eux et avait un impact sur la relation médecin-malade pour 27 (83,9\%) d'entre eux. Malgré l'intérêt déclaré pour la thématique, de nombreux médecins estimaient que le fait d'avoir un patient socialement vulnérable n'influençait pas leur prise en charge. À partir de ces données quantitatives, cette affirmation peut refléter de nombreuses significations. L'hypothèse d'une certaine forme de préoccupation pour l'égalité devant les soins, voire de revendication de cette égalité devant les soins, peut être formulée. Néanmoins, cela montre également que le concept d'équité des soins était insuffisamment pris en compte par les médecins généralistes participants. Un travail de recherche qualitatif apporte des données complémentaires confirmant cette hypothèse: les médecins interviewés au cours de ce travail manifestaient une volonté de soins identiques pour tous, tout en exprimant une certaine forme d'ambivalence quant à l'adaptation effective de la prise en charge dans des contextes socio-économiques difficiles (ErntsToulouse, 2014).

\section{2) Population des patients inclus dans l'étude}

Au total, 217 patients ont été sollicités pour participer à cette étude. Dix d'entre eux ont refusé de participer. Le taux de participation, de 95,4\%, était donc plutôt satisfaisant et permet de limiter un biais de sélection (notamment en défaveur des patients les plus socialement défavorisés). Associé au processus de randomisation, ce dernier permet de présupposer une certaine représentativité de l'échantillon ainsi constitué.

207 patients inclus, 60,4 \% étaient des femmes. L'âge moyen des participants était de 54,7 ans. Ces données sont concordantes avec les autres données disponibles sur les patients français rencontrés en médecine générale (Letrillart, 2014). Le détail des caractéristiques de notre échantillon est disponible dans le tableau 1 en annexe.

Concernant la précarité, 60 participants $(28,9 \%)$ avaient un score EPICES supérieur ou égal à 30, les classant ainsi en situation de précarité. Cette proportion est supérieure à celle attendue en population générale (ministère de l'Emploi et de la Solidarité, 1998 ; Robert, 2013). Étant donné l'état de moins bonne santé relatif des personnes précaires, 
il serait logique de constater une surreprésentation de ces dernières dans les cabinets de médecine générale. La confrontation de nos résultats à la littérature est limitée par le peu de données disponibles. En effet, des études ont permis d'évaluer la proportion de personnes précaires en centre de santé et en dispensaire, avec respectivement $39,4 \%$ et $45 \%$ des patients précaires selon le score EPICES (Blanquet, 2016; Merson, 2011). Or, ces centres concentrent une population plus défavorisée, ce qui explique cette proportion nettement plus importante (Afrite, 2011). Les autres estimations disponibles de la précarité en milieu de soins, effectuées à l'aide du score EPICES, ont été conduites en milieu hospitalier, avec un recrutement de patients très différent du nôtre. Les patients précaires représentaient dans ces différents travaux $23 \%$ des accouchées dans un service d'obstétrique (Convers, 2012), 46,4\% des patients d'un service d'endocrinologie-diabétologie (Bihan, 2012), et enfin, $57 \%$ des patients pris en charge dans un service dédié au diabète gestationnel dans un secteur très défavorisé (Cosson, 2015). La comparaison des résultats n'étant pas possible dans ces cas, nous ne pouvons qu'estimer la validité externe de nos résultats. Notre proportion de patients précaires semble ainsi concorder avec les données disponibles.

\section{3) Concordance entre score EPICES et repérage spontané des médecins généralistes}

Dans notre étude, le nombre de personnes définies comme précaires selon le score EPICES était de 60 (28,9\%). Sur les 147 patients non précaires selon le score EPICES, 126 (85,7 \%) l'étaient également selon l'évaluation spontanée du médecin généraliste. De la même manière, sur les 58 patients précaires selon le score EPICES, 31 (53,4 \%) l'étaient aussi après évaluation spontanée du médecin. Le détail de ce tableau de contingence est disponible dans le tableau 2 (situé en annexe). Le coefficient de corrélation Kappa de Cohen entre l'évaluation de la précarité par score EPICES et l'évaluation spontanée du médecin généraliste était de 0,404 ( $\mathrm{p}<0,01)$. Le score de corrélation Kappa de Cohen est exprimé par un nombre allant de -1 à 1 . Ce dernier correspond à une concordance parfaite et le zéro correspond à une corrélation imputable au hasard. La valeur relevée peut donc être considérée comme un accord faible à modéré selon Landis et Koch (Landis, 1977). Cette corrélation très modérée entre l'évaluation spontanée du médecin généraliste et du score EPICES permet d'illustrer la difficulté réelle d'identification des patients précaires par le médecin généraliste. Ces difficultés pratiques sont d'autant plus significatives qu'elles sont rencontrées par des médecins généralistes maitres de stage, ayant en moyenne de nombreuses années d'expérience professionnelle, s'exprimant au sujet de patients consultant dans leurs cabinets probablement depuis plusieurs années. Des études qualitatives ont permis l'identification de ces difficultés (Köhl, 2014). Ainsi, malgré sa proximité avec le patient, le médecin généraliste ressent des difficultés dans l'évaluation de la position sociale et ces difficultés se traduisent réellement par un repérage erroné dans de nombreux cas.

L'exploration des données produites par l'échelle de certitude a apporté des résultats originaux. En effet, le score de certitude moyen de l'évaluation effectuée par le médecin était de 7,7 (DS=2,3). Une association statistiquement significative entre l'évaluation positive de la précarité par le MSU et un score de certitude faible a été retrouvée $(\mathrm{p}<0,01)$. En d'autres termes, un médecin évaluant son patient comme précaire était significativement moins sûr de son évaluation, en évaluant sa certitude de 1 à 5 . Inversement, lorsqu'il estimait que son patient n'était pas précaire, il était 
significativement plus confiant dans son évaluation, et évaluait sa certitude sur la partie plus élevée de l'échelle (score de certitude de 6 à 10). Le tableau 3 en annexe expose les données détaillées relatives à ce point des résultats. Paradoxalement, le score de certitude n'était pas associé à une concordance (ou au contraire à une discordance) entre les estimations de la précarité par le score EPICES et par le médecin généraliste. Ce résultat met en évidence un point inattendu lors de la conception de l'étude. En effet, l'hypothèse était que le score de certitude serait meilleur dans les situations de concordance entre les deux évaluations. Contre toute attente, les médecins généralistes participants ont finalement associé certitude et absence de précarité et incertitude et précarité. Cette association soulève de nombreuses interrogations et nécessite d'être explorée par d'autres études, notamment qualitatives, pour expliquer ce phénomène. Néanmoins, il semble que la connotation péjorative du terme "précaire " ait poussé les médecins à se déclarer moins sûrs de leur « jugement ». Cette notion d'incertitude a déjà fait l'objet d'études dans le contexte de la médecine générale. Géraldine Bloy la considère comme indissociable, et même "constitutive ", de l'exercice de la médecine générale (Bloy, 2008). Ainsi, bien que confrontés de façon régulière (pour ne pas dire continue) à l'incertitude dans le domaine biomédical, les médecins généralistes semblaient peu familiers avec cette notion de précarité, qui semble revêtir une connotation trop péjorative pour l'affirmer. Des études mettant en évidence une certaine forme d'adhésion des médecins aux stéréotypes viennent renforcer cette hypothèse (Köhl, 2014 ; Lemaire-Lesoin, 2012 ; Van Ryn, 2000). Certains auteurs rapprochent la notion d'incertitude et la notion de gut feeling (pouvant être traduite par "sentiments viscéraux»). Selon eux, cette notion véhiculerait deux sentiments mis en jeu dans certaines prises en charge biomédicales : le sense of alarm (sens de l'alarme) et le sense of reassurance (sens de la réassurance) (Gelly, 2014 ; Lorenzo, 2013 ; Stolper, 2009). Ce gut felling est décrit soit comme une impression ressentie par le praticien de situation grave, alors qu'il n'a aucun élément objectif pour étayer cette sensation, soit comme une impression de situation bénigne alors qu'aucun diagnostic ou examen objectif ne permet de l'affirmer. Ces sentiments sont considérés comme le fruit d'une intériorisation intuitive de nombreux facteurs très variés, et se rapprochent effectivement du repérage de la précarité.

Dans la mesure où la concordance entre les repérages est au mieux modérée et que le score de certitude n'est pas associé à un repérage efficient, les éléments permettant le repérage de la précarité soulèvent des interrogations. Dans notre étude, les variables associées, en analyse univariée, à une bonne ou mauvaise concordance entre le score EPICES et le repérage spontané de la précarité par les médecins généralistes sont présentées dans le tableau 4 (situé en annexe). Après une analyse multivariée, effectuée par régression logistique pas à pas, aucune variable n'était associée à la concordance entre les évaluations par score EPICES et par le médecin généraliste. En d'autres termes, aucune variable n'était donc associée à un repérage " correct » ou " incorrect » de la précarité. Il n'a pas été possible, pour des raisons éthiques et pratiques, d'évaluer l'aspect physique et vestimentaire des patients et donc son association à un bon repérage de la précarité. Toutefois, cette notion de présentation générale à l'autre (aspect vestimentaire, hygiène, façon de se tenir, etc.) est très présente dans les études qualitatives (Köhl, 2014).

32 Des études qualitatives ont permis de mettre en évidence une certaine forme de passivité dans le recueil des informations, une intuitivité et une absence de formalisation du recueil des données socio-économiques par les médecins (Ernts- 
Toulouse, 2014). Toujours selon ces études, les connaissances des médecins généralistes sur la vulnérabilité sociale de leurs patients étaient souvent empiriques et intuitives (Köhl, 2014 ; Lemaire-Lesoin, 2012). Une étude récente a démontré que la connaissance par les médecins généralistes des facteurs non biomédicaux pertinents pour une prise en charge suivait un gradient socialement différencié : les médecins connaissent mieux les caractéristiques socio-économiques de leurs patients les plus favorisés (Casanova, 2018). Ainsi, l'absence de bagage théorique des médecins généralistes sur ces notions socio-économiques pourrait induire une absence de systématisation dans le recueil de ces données, et donc une utilisation consciente et inconsciente non efficiente de ces dernières, contrairement aux données purement biomédicales.

\section{Conclusion}

Pour conclure, notre étude a permis de mettre en évidence une concordance faible à modérée entre le repérage de la précarité spontané et le repérage par score EPICES. Ces résultats illustrent les difficultés auxquelles les médecins généralistes sont confrontés dans la prise en compte de facteurs sociaux dans leur contexte de soins. Ces difficultés, dont les causes sont à explorer, pourraient être surmontées par l'utilisation systématiques d'outils comme le score EPICES.

\section{BIBLIOGRAPHIE}

Adams J. (2005), « How accurate are Townsend Deprivation Scores as predictors of self-reported health? A comparison with individual level data ", Journal of Public Health, vol. 27, n 1, p. 101-106.

Afrite A. (2011), « Les personnes recourant aux 21 centres de santé de l'étude Epidaure-CDS sontelles plus précaires? »BEH, $\mathrm{n}^{\circ} 165$.

Barbier J.-C. (2005), « La précarité, une catégorie française à l'épreuve de la comparaison internationale », Revue française de sociologie, vol. 46, n², p. 351.

Berkhout C. (2017), «Comparaison des exercices des médecins généralistes maîtres de stage universitaires et non-maîtres de stage en France », Exercer, n ${ }^{\circ}$ 135, p. 308-309.

Bihan H. (2012), « Screening for deprivation using the EPICES score: a tool for detecting patients at high risk of diabetic complications and poor quality of life ", Diabetes \& Metabolism, vol. 38, $\mathrm{n}^{\circ} 1$, p. 82-85.

Blanquet M. (2016), « Metabolic syndrome and social deprivation: results of a French observational multicentre survey », Family Practice, vol. 33, n 1, p. 17-22.

Bloy G. (2008), « L'incertitude en médecine générale : sources, formes et accommodements possibles ", Sciences sociales et santé, vol. 26, n 1, p. 67.

Carstairs V. (1989), « Deprivation and mortality: an alternative to social class? », Community Medicine, vol. 11, $n^{\circ}$ 3, p. 210-219. 
Casanova L. et al. (2018), «Level of agreement between physician and patient assessment of nonmedical health factors ", Family Practice, vol. 35, n 4, p. 488-494.

Castiel D. (2009), « Handicap social et hôpitaux publics : pour un GHS "socialisable" ", La Presse Médicale, vol. 38, n 1, p. 142-145.

Cingolani P. (2006), La précarité, Paris, Presses universitaires de France.

Collet M. (2001), Dynamiques de précarisation, modes d'adaptation identitaire et interactions avec les logiques de santé, mémoire de DEA de sociologie, université Paris 8.

Convers M. (2012), «Intérêt de l'échelle d'évaluation de la précarité EPICES en obstétrique », Gynécologie Obstétrique \& Fertilité, vol. 40, n 4, p. 208-212.

Cosson E. (2015), « Psychosocial deprivation in women with gestational diabetes mellitus is associated with poor fetomaternal prognoses: an observational study », BMJ Open, vol. $5, \mathrm{n}^{\circ} 3$.

Ernts-Toulouse C. (2014), « Recueil de la situation sociale des patients et prise en charge des inégalités sociales de santé : perspectives en médecine générale ", université de Nantes, thèse d'exercice.

Eroglu S. (2007), « Developing an Index of Deprivation Which Integrates Objective and Subjective Dimensions: Extending the Work of Townsend, Mack and Lansley, and Halleröd ", Social Indicators Research, vol. 80, n 3, p. 493-510.

Ministère de l'Emploi et de la Solidarité (1998), « La progression de la précarité en France et ses effets sur la santé ", Rennes, Éditions ENSP.

Furtos J. (2000), « Épistémologie de la clinique psychosociale (la scène sociale et la place des psy) », Précarité et troubles psychiques, Quelle politique ? vol. 46, n 1, p. 23-32.

Furtos J. (2007), « Les effets cliniques de la souffrance psychique d'origine sociale », Mentalidées, vol. 11, p. 24-33.

Gelly J. (2014), « Tolérance à l'incertitude en médecine générale », Exercer, n 112 supplément 1, p. S6-S7.

Jordan H. (2004), « The Index of Multiple Deprivation 2000 and accessibility effects on health », Journal of Epidemiology \& Community Health, vol. 58, $n^{\circ}$ 3, p. 250-257.

Kohl M. (2014), « Les représentations de la précarité chez les médecins généralistes. Étude qualitative ", université de Bourgogne, thèse d'exercice.

Labbe E. (2015), « A new reliable index to measure individual deprivation: the EPICES score ", European Journal of Public Health, vol. 25, $\mathrm{n}^{\circ}$ 4, p. 604-609.

Labbe E. (2007). " Un indicateur de mesure de la précarité et de la "santé sociale" » : le score EPICES : L'expérience des Centres d'examens de santé de l'Assurance maladie », La Revue de l'Ires, vol. $53, \mathrm{n}^{\circ} 1$.

Landis J. R. (1977). « The measurement of observer agreement for categorical data », Biometrics, vol. $33, \mathrm{n}^{\circ} 1$, p. 159-174.

Lemaire-Lesoin C. (2012), « Représentations des médecins généralistes sur les inégalités sociales de santé », université Joseph Fournier, thèse d'exercice.

Letrillart L. (2016), « Comparison of French training and non-training general practices: a crosssectional study », BMC Medical Education, vol. 16, $\mathrm{n}^{\circ} 1$. 
Letrillart L. (2014), «ECOGEN. Étude des Éléments de la COnsultation en médecine GENérale », Exercer, vol. 114, p. 148-157.

Littre É. (1880), Dictionnaire de la langue française, Paris, Hachette.

Lorenzo M. (2013), « Tolérance à l'incertitude en médecine générale », université de Strasbourg, thèse d'exercice.

Merson F. (2011), « Précarité sociale et perception du temps, impact sur le sevrage tabagique », Santé Publique, vol. 23, n 5, p. 359.

Ministère de l'Emploi et de la Solidarité (1998), « La progression de la précarité en France et ses effets sur la santé », Rennes, Éditions ENSP.

Ministère des Affaires sociales et de l'Intégration (1992), « Arrêté du 20 juillet 1992 relatif aux examens périodiques de santé », Journal officiel n² 218, 19 septembre 1992.

Moulin J. J. (2005), « Le score EPICES : l'indicateur de précarité des Centres d'examens de santé de l'Assurance Maladie », rapport d'étude, Saint-Étienne, Centre technique d'appui et de formation des centres d'examens de santé, p. 18.

Moulin J. J. (2006), « Précarité et facteurs de risque : le score EPICES », Revue Francophone de Psycho-Oncologie, vol. 5, n 2, p. 115-121.

Pascal J. (2006), « Peut-on identifier simplement la précarité sociale parmi les consultants de l'hôpital ?", Sciences sociales et santé, vol. 24, n³ 3, p. 33-58.

Robert S. (2013), « Construction et validation d'un score individuel de précarité utilisable en soins primaires : une étude à partir de la cohorte santé, inégalités et ruptures sociales ", université Pierre et Marie Curie - Paris VI, thèse d'exercice.

Salmond C. (2006), « NZiDep: A New Zealand index of socioeconomic deprivation for individuals », Social Science \& Medicine, vol. 62, n 6, p. 1474-1485.

Sass C. (2006a), « Comparison of the individual deprivation index of the French Health Examination Centres and the administrative definition of deprivation », Santé Publique, vol. 18, $\mathrm{n}^{\circ} 4$, p. 513-522.

Sass C. (2006b), « Le score Epices : un score individuel de précarité. Construction du score et mesure des relations avec des données de santé, dans une population de 197389 personnes », BEH, vol. 14, p. 93-96.

Stolper E. (2009), « The diagnostic role of gut feelings in general practice. A focus group study of the concept and its determinants ", BMC Family Practice, vol. 10, $n^{\circ} 1$.

Van Ryn M. (2000), « The effect of patient race and socio-economic status on physicians' perceptions of patients ", Social Science \& Medicine, vol. 50, n 6, p. 813-828.

Vaucher P. (2012), « Detecting and measuring deprivation in primary care: development, reliability and validity of a self-reported questionnaire: the DiPCare- $Q$ », BMJ Open, vol. 2, $\mathrm{n}^{\circ} 1$.

Wrezinski J. (1987), « Grande pauvreté et précarité économique et sociale », rapport présenté au Conseil économique et social français les 10 et 11 février 1987.

\section{ANNEXES}


Tableau 1. Caractéristiques de l'échantillon

\begin{tabular}{|c|c|c|}
\hline & Effectif & $\%$ \\
\hline Personne précaire selon le score EPICES & 60 & 28,9 \\
\hline Sexe féminin & 125 & 60,4 \\
\hline Situation matrimoniale & & \\
\hline Vie en couple & 135 & 65,2 \\
\hline Vie seule & 72 & 34,8 \\
\hline Niveau d'études & & \\
\hline Inférieur au baccalauréat & 123 & 59,4 \\
\hline Niveau Baccalauréat & 56 & 27,1 \\
\hline Supérieur au baccalauréat & 28 & 13,5 \\
\hline Profession et catégorie sociale & & \\
\hline Agriculteurs exploitants & 1 & 0,5 \\
\hline Artisans, commerçants, chefs d'entreprise & 3 & 1,4 \\
\hline Cadres et professions intellectuelles supérieures & 5 & 2,4 \\
\hline Professions intermédiaires & 9 & 4,3 \\
\hline Employés & 56 & 27,1 \\
\hline Ouvriers & 13 & 6,3 \\
\hline Retraités & 86 & 41,5 \\
\hline Autres personnes sans activité professionnelle & 24 & 11,6 \\
\hline Pérennité de l'emploi & & \\
\hline Emploi non pérenne & 22 & 22,7 \\
\hline Emploi pérenne & 75 & 77,3 \\
\hline
\end{tabular}

Tableau 2. Corrélation entre le score EPICES et l'évaluation spontanée de la précarité par les médecins généralistes maîtres de stage

\begin{tabular}{|c|c|}
\hline \multicolumn{2}{|c|}{ Évaluation selon le score EPICES } \\
\hline Non précaire & Précaire \\
\hline
\end{tabular}




\begin{tabular}{|l|l|l|l|l|}
\cline { 3 - 4 } \multicolumn{2}{|c|}{} & $n(\%)$ & $n(\%)$ & \\
\hline \multirow{2}{*}{ Estimation du médecin généraliste } & Non précaire & $126(85,7)$ & $27(46,5)$ & $<0,01$ \\
\cline { 2 - 5 } & Précaire & $21(14,3)$ & $31(53,5)$ & \\
\hline
\end{tabular}

Tableau 3. Étude du score de certitude de l'évaluation fournie par les médecins généralistes participants

\begin{tabular}{|l|l|l|l|l|}
\hline \multicolumn{2}{|l|}{} & \multicolumn{2}{l}{$\begin{array}{l}\text { Score de certitude de } \\
\text { l'évaluation }\end{array}$} & \\
\cline { 3 - 6 } & & 1 à 5 & 6 à 10 & Total \\
\cline { 3 - 6 } & & n (\%) & n (\%) & \\
\hline \multirow{2}{*}{$\begin{array}{l}\text { Évaluation de la précarité selon le } \\
\text { médecin généraliste }\end{array}$} & $\begin{array}{l}\text { patients non } \\
\text { précaires }\end{array}$ & $24(52,2)$ & $129(81,1)$ & 153 \\
\cline { 2 - 7 } & patients précaires & $22(47,8)$ & $30(18,9)$ & 52 \\
\hline Total & & 46 & 159 & 205 \\
\hline
\end{tabular}

Tableau 4. Analyse des variables associées à la concordance des évaluations des médecins généraliste et du score EPICES (analyse univariable)

\begin{tabular}{|l|l|l|l|}
\hline & Concordance & Discordance & $\mathbf{p}$ \\
\hline & $\mathrm{n}(\%)$ & $\mathrm{n}(\%)$ & \\
\hline Sexe féminin & $95(76)$ & $28(22,40)$ & $<0,01$ \\
\hline Situation matrimoniale & & & $<0,01$ \\
\hline Vie en couple & $105(77,78)$ & $29(21,48)$ & \\
\hline Vie seule & $52(72,22)$ & $19(26,39)$ & \\
\hline Niveau d'études & & & $<0,01$ \\
\hline Inférieur au baccalauréat & $89(72,36)$ & $32(26,02)$ & \\
\hline Niveau Baccalauréat & $45(80,36)$ & $11(19,64)$ & \\
\hline Supérieur au baccalauréat & $23(82,14)$ & $5(17,86)$ & \\
\hline Profession et catégorie sociale & & & 0,83 \\
\hline Agriculteurs exploitants & $0(0)$ & $1(100)$ & \\
\hline
\end{tabular}




\begin{tabular}{|c|c|c|c|}
\hline Artisans, commerçants, chefs d'entreprise & $10(76,92)$ & $3(23,08)$ & \\
\hline Cadres et professions intellectuelles supérieures & $4(80)$ & $1(20)$ & \\
\hline Professions intermédiaires & $9(100)$ & $0(0)$ & \\
\hline Employés & $43(76,79)$ & $12(21,43)$ & \\
\hline Ouvriers & $9(69,23)$ & $4(30,77)$ & \\
\hline Retraités & $66(76,74)$ & $19(22,09)$ & \\
\hline Autres personnes sans activité professionnelle & $16(66,67)$ & $8(33,33)$ & \\
\hline Pérennité de l'emploi & & & 0,05 \\
\hline Emploi non pérenne & $12(54,55)$ & $9(40,91)$ & \\
\hline Emploi pérenne & $63(84)$ & $12(16)$ & \\
\hline Patients bénéficiaires de la $\mathrm{CMU}$ & $12(66,67)$ & $6(33,33)$ & $<0,01$ \\
\hline Patients bénéficiaires d'une mutuelle & $145(76,32)$ & $43(22,63)$ & $<0,01$ \\
\hline
\end{tabular}

\section{RÉSUMÉS}

Cette étude se propose d'étudier la concordance entre un repérage de la précarité effectué spontanément par les médecins généralistes et un repérage effectué par le score EPICES (évaluation de la précarité et des inégalités de santé dans les centres d'examens de santé), développé et validé dans les centres d'examens de santé.

Les 32 médecins généralistes ont permis d'inclure 207 patients dans l'étude. Le coefficient de concordance Kappa de Cohen était de 0,404, soit une corrélation faible à modérée.

L'étude des variables associées à la concordance de ce repérage n'a permis de dégager aucune variable significativement associée à la concordance après une analyse multivariée.

Pour conclure, le repérage de la précarité par les médecins généralistes reste difficile, ce qui concorde avec les données qualitatives des études précédemment réalisées.

This study aims to assess concordance between detection of the precariousness spontaneously carried out by the general practitioners and a scoring assessment by the EPICES score (developed and validated in the centers of health examinations).

Thirty-two general practitioners allowed to include 207 patients in the study. Cohen's Kappa concordance coefficient was 0.404 , i.e. a low to moderate correlation.

The study of the variables associated with the concordance of both assessments did not show any variable significantly associated with concordance after mutlivariate analysis.

To conclude, the identification of precariousness by general practitioners remains difficult, which is consistent with the qualitative data from previous studies. 
INDEX

Mots-clés : précarité, déterminants sociaux de la santé, médecin généraliste, soins premiers

Keywords : precariousness, social derterminants of heatlh, general practitionners, primary healthcare

\section{AUTEURS}

\section{KATIA MAZALOVIC}

Université de Bourgogne, LIR3S-UMR 7366, Département de Médecine générale, UFR Santé

\section{CLAIRE ZABAWA}

Université de Bourgogne, Département de Médecine générale, UFR Santé

\section{PIERRE-HENRI ROUX-LEVY}

Université de Bourgogne, Département de Médecine générale, UFR Santé

\section{MARYSE GAIMARD}

Professeure de démographie

Université de Bourgogne, LIR3S-UMR 7366

maryse.gaimard@u-bourgogne.fr 